\title{
1 EFFECT OF HANDLING ON NEUROTRANSMITTER PROFILE IN PIG BRAIN \\ 2 ACCORDING TO FEAR RELATED BEHAVIOUR
}

3

4 Laura Arroyo ${ }^{\mathrm{a}}$, Ricard Carreras ${ }^{\mathrm{b}}$, Daniel Valent ${ }^{\mathrm{a}}$, Raquel Peña ${ }^{\mathrm{a}, \mathrm{c}}$, Eva Mainau ${ }^{\mathrm{d}}$, Antonio

$5 \quad$ Velarde $^{\mathrm{b}}$, Josefa Sabriàe ${ }^{\mathrm{e}}$, Anna Bassols ${ }^{\mathrm{a}, \mathrm{c}}$

6

7

8 aDepartament de Bioquímica i Biologia Molecular, Facultat de Veterinària. Universitat

9 Autònoma de Barcelona, 08193 Cerdanyola del Vallès, Spain

' IRTA, Animal Welfare Subprogram, Veïnat de Sies, s/n, 17121 Monells, Spain

cServei de Bioquímica Clínica Veterinària, Facultat de Veterinària. Universitat Autònoma de Barcelona, 08193 Cerdanyola del Vallès, Spain

${ }^{\mathrm{d}}$ Departament de Ciència Animal i dels Aliments, Facultat de Veterinària. Universitat 14 Autònoma de Barcelona, 08193 Cerdanyola del Vallès, Spain

eDepartament de Bioquímica i Biologia Molecular, Facultat de Medicina. Institut de

Corresponding author: Anna Bassols

anna.bassols@uab.cat

20

Departament de Bioquímica i Biologia Molecular, Facultat de Veterinària. Universitat

Autònoma de Barcelona, 08193 Cerdanyola del Vallès, Spain

\footnotetext{
"This is an accepted manuscript of an article published by Elsevier in "Phisiology \& Behavior", vol. 167 (December 2016), p. 374-381.Available at: http://dx.doi.org/10.1016/j.physbeh.2016.10.005"

(C) 2016. This manuscript version is made available under the CC-BY-NC-ND 4.0 license http://creativecommons.org/licenses/by-nc-nd/4.0/
} 


\section{ABSTRACT}

Chemical neurotransmitters (NT) are principal actors in all neuronal networks of animals. The central nervous system plays an important role in stress susceptibility and organizes the response to a stressful situation through the interaction of the dopaminergic and the serotonergic pathways, leading to the activation of the hypothalamus-pituitary-adrenal axis (HPA). This study was designed to investigate: a) the effects of stressful handling of pigs at the slaughterhouse on the neurotransmitter profile in four brain areas: amygdala, prefrontal cortex (PFC), hippocampus and hypothalamus, and b) whether the alterations in the brain NT profile after stressful handling were associated with fear, determined by the tonic immobility (TI) test. In the first place, the characterization of the NT profile allowed to distinguish the four brain areas in a principal component analysis. The most crucial pathway involved in the reaction of pigs to a stressful handling was the serotonergic system, and changes were observed in the amygdala with a decrease in serotonin (5-HT) and total indoleamines, and in the hippocampus, where this pathway was activated. Fearful and non-fearful pigs did not show significant differences in their NT profile in control conditions, but when subjected to a stressful handling in the slaughterhouse, fearful animals showed a significant variation in the serotonin pathway and, in a lesser extent, the dopamine (DA) pathway. In conclusion, the existence of an underlying biological trait - possibly fearfulness - may be involved in the pig's response toward stressful challenges, and the serotonergic system seems to play a central role in this response.

Keywords: brain, dopamine, pig, serotonin, stress, tonic immobility 


\section{Introduction}

Chemical neurotransmitters (NT) are principal actors in all neuronal operations. The noradrenergic, dopaminergic and the serotonergic pathways are the most important and well characterized systems underlying the response to stress, fear and reward, among others. The central nervous system controls the action of endocrine glands through the release of catecholamines, indoleamines and other transmitters which can be excitatory or inhibitory mediators [1]. Amygdala, hippocampus and prefrontal cortex (PFC) are recognized to play a role in the stress response organization. In these structures, stressors produce changes in extracellular concentrations of different NTs leading to activation and modulation of processes to cope with stress. These areas have an indirect output to the hypothalamus, which acts modulating the final stress response through the sympathetic nervous system and the activation of the hypothalamic-pituitary-adrenal (HPA) axis [2]. Therefore, the stress response involves not only the activity of these specific brain areas, but also the interaction among those areas through neuromodulators, especially catecholamines (noradrenaline (NA), dopamine (DA)) and the indoleamine serotonin (5-HT). DA is metabolized to homovanillic acid (HVA) and 3,4-dihydroxyphenyl acetic acid (DOPAC), whereas 5-HT is metabolized to 5-hydroxyindoleacetic acid (5-HIAA) [1]. Determining the ratios between the amine and its metabolites can indicate the turnover rate [3].

Tonic immobility (TI) is a well-established test to evaluate the fear response in a wide range of vertebrates and invertebrates [4,5]. Long duration of TI is generally considered as an indication for high levels of fearfulness associating tonic immobility with emotional components like fear or anxiety [6] and with a fear-related phenotype [7]. In pigs, the TI test has shown to be consistent with other behavioural tests carried out at different ages assessing fear, aggressiveness and behavioural strategies in front of a stressful situation, thus indicating that it may be related to individual personality characteristics [4,8-15]. A positive relationship 
has been reported between TI scores and lean meat percentage, and a genetic background has been suggested $[8,9]$. Furthermore, the fear-related behaviour is closely associated with the stress response regulated by the HPA axis $[7,16]$.

There are several stressors widely recognized and studied in pigs, such as handling, mixing, transport and slaughter [17]. One of the main consequences of pre-slaughter stress is the production of pale, soft and exudative (PSE) meat, leading to an organoleptic and economic cost [18]. In the literature, changes in brain NT profiles in genetically stress-susceptible pigs have been reported [19]. Immobilization of pigs produces changes in hypothalamic and/or hippocampal bioamine levels, suggesting an important role of these regions in the responsiveness of the pig to acute stress conditions [3,20]. Furthermore, the involvement of central nervous system NT in aggressiveness and dominance has also been studied [21-24]. However, changes in brain NT related to standard or commercial stress conditions at slaughter and the fear-related behaviour have been rarely studied in pigs [25].

In the present study, we have first characterized the NT profile of catecholamines and indoleamines in four different brain areas of the pig involved in the stress and fear response: amygdala, PFC, hippocampus and hypothalamus. Secondly, we have analysed the changes in NT profile in pigs subjected to stress at slaughter classified according to a fear-related phenotype.

\section{Materials and Methods}

\subsection{Animals, housing conditions, general procedure and ethical statement}

This study was carried out at the IRTA-Monells experimental farm (Monells, Spain). Ninetytwo male piglets were randomly allocated in 10 housing groups of 10-12 piglets each in the pre-control building at 3 weeks of age (mean \pm SE: $5.85 \pm 0.166 \mathrm{Kg}$ ). All piglets came from 
the same commercial farm and were crosses of Large White $\times$ Landrace Halothane gene RYR(1)- free (NN) sows with Pietrain heterozygous (Nn) terminal sire. At 4 weeks of age, all piglets were subjected to a TI test in order to select a total of 36 piglets (18 positive to TI and 18 negative to TI, see 2.2). At 8 weeks of age, pigs were moved to the control building and randomly allocated in four groups of nine.

Each group was housed in slatted pens $(5 \mathrm{~m}$ x $2.70 \mathrm{~m})$ under natural light conditions at a constant environmental temperature of $22 \pm 3{ }^{\circ} \mathrm{C}$. Each pen was provided with one steel drinker bowl $(15 \mathrm{~cm}$ x $16 \mathrm{~cm})$ connected to a nipple and a concrete feeder $(58 \mathrm{~cm} \mathrm{x} 34 \mathrm{~cm})$ with 4 feeding places. Pigs had water and food ad libitum. The pigs were inspected daily and no health problems were observed during the experimental period. The study was approved by the Institutional Animal Care and Use Committee (IACUC) of IRTA.

\subsection{Tonic immobility test}

Piglets were subjected to a TI test adapted from Erhard et al. [10] and de Sevilla et al. [8]. An experimenter restrained individually each piglet in a dorsal decubitus position using a Vshaped wooden restrain (50 cm long and with an $80^{\circ}$ angle). Another experimenter placed a small bag ( $15 \mathrm{~cm} \times 20 \mathrm{~cm}$ and weighing $500 \mathrm{~g})$ over the piglet's throat with one hand, while carefully holding the hind legs with the other until the animal remained immobile. Only one induction was performed and the time between the experimenter's hands were removed from the animal's hind legs and the time that the piglet tried to turn was recorded. If the piglet did not try to turn within $3 \mathrm{~min}$, the trial finalized, and the time of $180 \mathrm{~s}$ was assigned to this piglet. Otherwise, piglets that did not show the immobility response because they struggled while they were being placed onto the V-shaped wooden restrain were assigned a time of $0 \mathrm{~s}$. The 18 piglets with the lowest time (less than or equal to $10 \mathrm{~s}$ ) to try to turn were chosen and classified as negative to TI test and the 18 piglets with the highest time (equal to or more than 
54 s) to try to turn were also selected and classified as positive to TI test. An outline of the experimental design and the distribution of TI negative and TI positive animals is shown in Figure 1.

\subsection{Housing and slaughtering conditions.}

Animals aged 24 weeks were fasted $8 \mathrm{~h}$ before being transported from the experimental farm to the experimental slaughterhouse (1.2 km of distance). "Control" and "stress" conditions included different management during unloading, lairage and conduction to the stunning area. During the unloading, the pigs of two housing pens (9 TI-negative, 9 TI-positive) were subjected to stress by noise, human presence and rough handling (simulating commercial conditions) whereas the pigs of the other two housing pens (9 TI-negative, 9 TI-positive) were handled very calmly allowing the time need for the animals to go ahead by themselves. Pigs were located in the lairage pens for an hour, the 18 animals under stressful conditions were mixed between the two housing groups, whereas the 18 animals under control conditions remained separated maintaining the housing groups. The management during the conduction of the pigs to the stunning area was similar to the management during unloading (control or stress). The total length of the procedure was approximately $90 \mathrm{~min}$. Animals were stunned in groups of two by exposure to $90 \% \mathrm{CO}_{2}$ at atmospheric air for 3 min and exsanguinated afterwards.

\subsection{Tissue sampling and neurotransmitter quantification.}

Immediately after the slaughter $(\approx 5 \mathrm{~min})$ the skull was opened. The brain was removed and tissue samples from the selected brain structures (amygdala, PFC, hippocampus and hypothalamus) were excised, collected as quickly as possible (within $90 \mathrm{~s}$ ) in liquid $\mathrm{N}_{2}$ and kept frozen at $-80^{\circ} \mathrm{C}$, until NT analysis according to a procedure adapted from Sabrià et al. [26]. Samples were weighted and homogenized $(1: 10 \mathrm{w} / \mathrm{v})$ in ice-cold $0.25 \mathrm{M}$ perchloric acid 
containing $0.1 \mathrm{M} \mathrm{Na}_{2} \mathrm{~S}_{2} \mathrm{O}_{5}$ and $0.25 \mathrm{M}$ ethylenediaminetetraacetate (EDTA).

Dihydroxybenzylamine (DHBA) and $\mathrm{N} \omega$-metil-5-hydroxytryptamine $(\mathrm{N} \omega)$ were added as

internal standards for catecholamines and indoleamines, respectively. The mixtures were

homogenized by sonication (Branson Digital Sonifier, model 250, Branson Ultrasonics Corp.,

Danbury, CT) followed by centrifugation at $3000 \mathrm{~g}$ for $10 \mathrm{~min}$ at $4{ }^{\circ} \mathrm{C}$ and the supernatants

were kept frozen at $-80^{\circ} \mathrm{C}$. After centrifugation at $12000 \mathrm{~g}$ for $10 \mathrm{~min}$ at $4^{\circ} \mathrm{C}$, the

concentration of catecholamines (NA, DA, DOPAC and HVA) and indoleamines (5-HT and

5-HIAA) were determined in $20 \mu \mathrm{L}$ aliquots using HPLC (Elite LaCHrom, Merck, Hitachi, Japan) equipped with a Chromolith Rp-18e 100 x 4.6 mm column (Merck KgaA, Darmstadt,

Germany) with electrochemical detection (ESA Coulochem II 5200, Bedford, MA). The

mobile phase consisted of $0.5 \mathrm{M}$ citrate buffer $\mathrm{pH}$ 2.8, $0.05 \mathrm{mM}$ EDTA, $1.2 \mathrm{mM}$ sodium octyl sulphate (SOS) and 1\% acetonitrile. The applied voltage was set at $400 \mathrm{mV}$ and the flow rate was $1 \mathrm{~mL} / \mathrm{min}$.

The chromatographic quantification of dopaminergic and serotonergic NTs showed a good precision, with coefficient of variation between-days and within-days lower than $4 \%$. HVA and $2.5-120 \mathrm{pg} / \mu \mathrm{l}$ for the rest of NTs. Coefficients of determination $\left(\mathrm{R}^{2}\right)$ were calculated and found to be higher than 0.999 for all analytes. Limit of detection was between 2.14 and $4.97 \mathrm{pg} / \mu \mathrm{L}$ and the limit of quantification was between 6.48 and $15.06 \mathrm{pg} / \mu \mathrm{L}$ for all the analytes. The internal controls (DHBA and $\mathrm{N} \omega$ ) allowed the comparison between runs.

Total content of catecholaminergic and serotonergic pathways and ratios of DOPAC and HVA to DA, and 5-HIAA to 5-HT were estimated as a measure of DA and 5-HT turnover or rate metabolism in these brain regions.

\subsection{Statistical analysis}


The statistical analysis was carried out with the Statistical Analyses System (SAS V9.2; software SAS Institute Inc., Cary, NC; 2002-2008). The significance level was established at $P<0.05$ and a tendency was considered at $0.05 \leq P \leq 0.1$. Descriptive data are presented with the means and the standard error (mean $\pm \mathrm{SE})$.

Whenever possible, data was log transformed to correct the distribution and hence permit use of parametric statistics. Normality test of data and residuals was performed for each measure. Normally distributed measures were analyzed using the MIXED procedure of SAS with Tukey adjustment. Measures with Poisson or multinomial distributions were analyzed using the GENMOD procedure of SAS. In all models, each pig was introduced as the experimental unit, the fixed effects included were type of handling and immobility test and planned pairwise comparisons with Bonferroni correction were performed.

Factor analysis: Interrelations among the seven neurotransmitters were included in a common factor analysis using principal component solution (PCA) to identify unobserved common factors that explain differences between regions. The criteria used to determine the number of factors to retain were: (a) eigenvalues $>1$ and (b) total variance accounted greater than $60 \%$. After the initial factor extraction, the matrix was orthogonally rotated (varimax method) to maintain factors independent and uncorrelated. Thus, each variable had a high loading (correlation coefficient between variables and factors) on a single factor and a small or moderate loading on other factors, using 0.5 loading in absolute value as cut-off point to accept a variable into a factor.

Each brain region of each pig obtained an individual score on each factor. Factor scores were normally distributed with a mean of zero.

\section{Results}




\subsection{Tonic Immobility test}

189

190

191

192

The mean time of the 92 piglets to turn was $34.80 \pm 3.77$ s. Five of the 92 piglets $(5.43 \%)$ did not show immobility response therefore they were classified as negative to TI. Three of the 92 animals (3.26\%) did not turn during the $3 \mathrm{~min}$ of the test and were classified as positive to TI. Since this was not enough to build groups with the required sample size, the 18 individuals with the most extreme behaviours were chosen. The mean time of the animals negative to TI and positive to TI was $5.00 \pm 0.93 \mathrm{~s}$ and $93.05 \pm 10.22 \mathrm{~s}$, respectively, thus both groups were considerably apart (Figure 1). Long duration of TI is considered as an indication for high levels of fearfulness, thus negative pigs to TI were classified as non-fearful animals and positive pigs as fearful animals

\subsection{Levels of brain amines and their metabolites in amygdala, PFC, hippocampus} and hypothalamus.

Table 1 shows the regional distribution of catecholamines and indoleamines in the four brain regions. Highest concentrations of NA were found in the hypothalamus. The concentration of DA and its metabolites DOPAC and HVA were found to be highest in the amygdala and hypothalamus. The ratio DOPAC/DA and HVA/DA was highest in the PFC.

Regarding to indoleamines, the highest concentration of 5-HT was found in the amygdala and hypothalamus, whereas the ratio 5-HIAA/5-HT was similar in all structures.

Principal Component analysis (PCA) reduced the seven variables (NA, L-DOPA, DOPAC, DA, HVA, 5-HIAA and 5-HT) to 2 common factors or principal components explaining 92.11 $\%$ of the variance. The eigenvalues, the individual and cumulative percentage accounted and the varimax rotated factor loadings for each variable are shown in Table 2. Brain regions with 
a high score for factor 1 (PC1) had high levels of DOPAC, DA, HVA, 5-HIAA and 5-HT; and brain regions with a high score for factor 2 (PC2) had high levels of NA and L-DOPA.

The PCA score plot showed that the pattern of NTs was able to readily differentiate all four brain areas (Figure 2).

\subsection{Influence of handling stress at slaughter on brain NTs.}

The concentrations of brain monoamines in the amygdala, PFC, hippocampus and hypothalamus are presented in Table 3.

The handling stress group presented lower concentration of 5-HT $(P=0.044)$, HVA $(P=$ $0.028)$ and a tendency for DA $(P=0.064)$ in the amygdala. As a consequence, a decrease in total indole content was observed in this area $(P=0.043)$. In the hippocampus, the concentration of 5-HIAA $(P=0.031), 5$-HT $(P=0.054$, tendency $)$ and total indole content $(P$ $=0.024)$ was found to be higher in animals exposed to handling stress. Catecholamine levels did not show difference between handling groups, but an increase in the ratio indole/catecholamine $(P=0.012)$ was found in this area. In the hypothalamus, an increase in HVA $(P=0.017)$ and in the sum of the metabolites DOPAC + HVA $(P=0.020)$ was observed in stressed pigs. Finally, no difference in any monoamine and their metabolites was found in the PFC.

To find out whether the fearful individuals (TI positive) showed a higher response to a stressful situation in the slaughterhouse, pair-wise comparisons were performed within handling groups (Table 4). Indeed, there were no differences in the NT profile between fearful and non-fearful groups in the control situation. In contrast, significant differences were found between TI positive and TI negative groups when stressfully handled at the slaughterhouse. 
tendency for total indoleamines $(P=0.063)$ in the hippocampus and a tendency to increase LDOPA $(P=0.090)$ in the hypothalamus compared to non-fearful animals.

\section{Discussion}

Following classical neurology, the neural pathways controlling response to stress, fear, aggression, emotion, decision-making and other behaviours are allocated in specific brain areas such as the amygdala, the hippocampus and the PFC [27-32]. They process sensory information to organize the autonomic response to stimuli from the environment or from internal cues and, in particular, these areas are involved in the control of stress and the regulation of the HPA axis [33]. Catecholaminergic (NA, DA and their metabolites), and serotonergic (5-HT and 5-HIAA) systems play a significant role in integrating the activity and interaction among those areas $[1,34]$.

In this work, we have shown that, together with the hypothalamus, these areas are characterized by a particular pattern of NT that clearly discriminate the four regions. In the PCA, component 2 (high concentrations of NA and L-DOPA) characterized the hypothalamus versus the other three areas, whereas the first component (high concentrations of the other NTs) characterized the amygdala versus PFC and hippocampus. These two areas were the most similar in their NT profile.

All three NT systems (noradrenergic, dopaminergic and serotonergic) have an important role in the control of the stress reaction [34]. Although, historically, the noradrenergic system in the locus coeruleus has attracted much attention in the study of the stress response, the dopaminergic and the serotonergic systems have also been consistently implicated [35]. In particular, 5-HT has remarkable modulatory effects in almost all central nervous system integrative functions, such as stress, mood, anxiety and aggression [36] and it has been recognized as being directly related to stress and able to regulate the HPA axis by stimulating 
$\mathrm{CRH}$ release in the paraventricular nucleus of the hypothalamus [37]. Marked changes in brain 5-HT turnover have been shown to occur in both rodents and humans upon activation of the HPA axis [38]. In particular, significant increases in the synthesis and release of 5-HT have been observed in various brain areas in response to different stressful conditions such as electrical foot shocks, cold environment, immobilization sessions, or tail pinches in rats [37]. All these data strongly support the existence of reciprocal relationships between the 5-HT system and the HPA axis.

The results presented here support the central role of the serotonergic pathway in the regulation of the short term reaction to acute stress in pigs. The most remarkable change induced by stressful handling at the slaughterhouse is the alteration of the serotonergic system in the hippocampus and in the amygdala. There is a decrease in the serotonin pathway (5-HT and total indoleamines, and a tendency for 5-HIAA) in the amygdala after acute handling stress. It is known that under stress conditions the locus coeruleus activates stress pathways in the amygdala through noradrenergic projections. Likewise, the amygdala sends projections to the hypothalamus and brain stem, mediating the unconscious acute responses to danger and orchestrating the expression of behavioural and physiological responses (e.g. changes in heart rate, respiration and pupillary dilation) [35]. Thus, the amygdala and the hypothalamus are connected to innate (unconditioned) fear and may serve to enhance the state of arousal in order to adapt to challenging situations [39]. A decrease in 5-HT in the amygdala has been shown in rats subjected to forced swimming as a model of acute stress [34], although contradictory results have been reported that are probably explained by the existence of specific regions inside the amygdala with different functional roles [27].

The hippocampus is central to 5-HT function since it receives a dense projection of 5-HT fibres mainly from the raphe nucleus and it is rich in various 5-HT receptor types, being a mediator in the relationship of 5-HT with the HPA axis [32,37]. The increase of 5-HT 
(tendency) as well as its metabolite 5-HIAA induced by stressful handling indicates that this NT is synthesized and rapidly metabolized. This is in agreement with the general idea that only inescapable, but not escapable, stresses produce an increase in extracellular 5-HT concentration in rat hippocampus [37,41]. In rodents, many studies have demonstrated that 5HT release is increased in the hippocampus during several stress conditions, including immobilization [42], psychological stress [43], exposure to cats, tail pinch and forced swimming [44] and footshock [45,46].

As stated above, the amygdala sends the distress signal to the hypothalamus, where an increase in HVA and HVA+DOPAC is observed, indicating a higher rate of DA catabolism. This indicates that the DA system, and not only the NA system, is activated by stressful stimuli, as suggested by others [35]. No changes in NA were detected in the hypothalamus in the present work, in contrast to the reported decrease in NA in this region in pigs that showed distressed behaviour at the slaughterhouse [25] and to acute immobilization stress [3,20], but their approach was different from our experimental setting.

$\mathrm{TI}$ is a measure of fear and this fear-related behaviour is closely associated with the stress response regulated by the HPA $[7,47,48]$. Due to this relationship, we analysed the alteration in NTs associated to the response to the TI test. To get a more accurate analysis of the NT response to fear, we analyzed the response of the animals to control or stressful handling at the slaughterhouse depending on their TI classification. Fearfulness could be considered a basic feature of the temperament of each individual, that predisposes it to respond to a variety of potentially alarming challenges [49].

The objective was to establish differences between individuals classified as having a fearrelated behaviour and those classified as non-fearful. Indeed, there were no significant differences between fearful and non-fearful animals in control conditions. In contrast, fearful 
animals displayed important changes in the NT profile in the stressful situation. Again, the serotonergic pathway was mostly affected, especially in the hippocampus. The hippocampus and the serotonergic system have been previously related to fear behaviour in pigs [50] and chicken [51]. In pigs, Ursinus et al. [50] recently reported that hippocampal 5-HT is positively correlated with standing alert time (freezing) and inversely correlated with locomotion and exploration in pigs subjected to a novel object test. Since freezing is a sign of fear and explorative behaviours are generally thought to reflect a low level of fear or anxiety, it is concluded that hippocampal 5-HT increases in a fear condition [52]. The authors did not find any relationship between behaviour in the novel object test and 5-HT levels in the PFC or in the hypothalamus. In agreement with these authors, our results support the hypothesis that the relations between behaviour and measures of 5-HT in brain indicate an underlying personality trait and that individual differences in behaviour of animals during environmental challenges may covary with the animal's serotonergic system functioning. It is also interesting to note that in Ursinus's work, hippocampal 5-HT activity measured at 19 weeks of age in euthanized animals was related to behaviours observed during the novelty test at 11 weeks of age [50]. Taken altogether, these results suggest that the hippocampus, but not other brain regions, might be involved in a putative personality measure in pigs related to the trait fearfulness, and that 5-HT would be the main neurotransmitter involved. In rats, a short lasting acute footshock session was able to induce a marked increase in 5-HT synaptic levels in the hippocampus as well as freezing and anxiety-related behaviours [46], and endogenous 5-HT seems to be responsible for the modulation of activity in the hippocampal pyramidal neurons linked to freezing behaviour [53]. Furthermore, mice with a genetic deletion of the serotonin 1A receptor (5-HT1AR) have been shown to be more fearful in a number of behavioural conflict tests, confirming the important role of this neurotransmitter and this receptor in modulating anxiety $[29,54]$. Thus, the role of the hippocampus and the serotonergic system in 
the fear-related responses to stressful challenges may be a general characteristic of animal species. Although our results indicate a principal role of 5-HT and the hippocampus, they also suggest the involvement of the catecholamine system, since total catecholamines are increased in this region.

It is interesting to speculate about the molecular changes that lead to variations in the NT concentration, taking into account that the methodological approach used in the present work measures the total amount of the NT. It is not possible in pigs to perform microdialysis experiments that would allow the direct measurement of extracellular NTs (presumably related to their presence at the synapsis) [55]. The mechanism involving NT release includes the synthesis of the NT by synthetic enzymes, their recruitment to vesicles and their release to the synaptic cleft [56]. Since we are measuring total content of NTs, rapid changes in total 5HT concentration could be related to a change of tryptophan hydroxylase 2 activity (TPH2), the enzyme that catalyzes the rate-limiting step in serotonin biosynthesis in the brain [57]. Current knowledge indicates that TPH2 is specifically transcribed in the somatodendritic segment of 5-HT neurons and a variable fraction of TPH2 mRNA is transported to terminal field [58]. A similar regulatory mechanism exist for tyrosine hydroxylase, the rate-limiting enzyme for the synthesis of catecholamines [59]. These enzymes can be rapidly transcribed in response to acute stress such as immobilization or other types of stress $[27,60]$. Other regulatory mechanisms that may be potentially involved are phosphorylation by protein kinase A (PKA) and the $\mathrm{Ca}^{2+} /$ calmodulin dependent protein kinase II [61-63] and proteinprotein interactions [59].

\section{Conclusions}

The most remarkable change induced by stressful handling is the alteration of the serotonergic system in the hippocampus and in the amygdala. There was no difference in neurotransmitter 
355 profile between fearful and non-fearful pigs when confronted to a non-stressful handling at

356 the slaughterhouse, but fearful animals did show more changes when subjected to stressful

357 handling, concerning specially the serotonergic pathway in the hippocampus.

358 In conclusion, the existence of an underlying biological trait - possibly fearfulness - may be

359 involved in pig's response toward stressful challenges, and the serotonergic system seems to 360 be central to this response. 
The authors declare that there is no conflict of interest associated with this manuscript.

\section{Acknowledgements}

We are grateful to Dr Mercedes Unzeta and Susana Benitez (Departament de Bioquímica i

368 Biologia Molecular. Facultat de Medicina, UAB) for their help with the HPLC analysis and

369 Anna Vilalta for her excellent technical assistance. This work was supported by grants

370 AGL2010-21578-C03-01 and AGL2011-30598-C03-02 from the Spanish Ministerio de

371 Economía y Competitividad, and grant 2014 SGR4 from the Generalitat de Catalunya. Part of

372 the funding was financed by the FEDER program from the European Union. 
376 Figure 1. Outline of the experimental design and the distribution of TI negative and TI

377 positive animals. Ninety-two pigs were subjected to the TI test with a maximum allowed time 378 of $180 \mathrm{~s}$. Three pigs did not turn (TI positive) and five moved immediately (time $=0 \mathrm{~s}$, TI

379 negative). The animals showing the most extreme responses in reaction time were selected to 380 be included in the study (18 animals for each group).

Figure 2. Score plot from a principal component analysis showing the distribution of the four 383 brain areas analysed (amygdala, PFC, hippocampus and hypothalamus) regarding their NT 384 profile. 


\section{REFERENCES}

[1] E.R. Kandel, J.H. Schwartz, T.M. Jessell, S.A. Siegelbaum, A.J. Hudspeth, Principles of neural science, 5th edition, McGraw-Hill Companies, Inc., 2013.

[2] F. Mora, G. Segovia, A. Del Arco, M. de Blas, P. Garrido, Stress, neurotransmitters, corticosterone and body-brain integration, Brain Res. 1476 (2012) 71-85.

[3] A.B. Piekarzewska, S.J. Rosochacki, G. Sender, The effect of acute restraint stress on regional brain neurotransmitter levels in stress-susceptible pietrain pigs, J. Vet. Med. Physiol. Pathol. Clin. Med. 47 (2000) 257-269.

[4] B. Forkman, A. Boissy, M.C. Meunier-Salaun, E. Canali, R.B. Jones, A critical review of fear tests used on cattle, pigs, sheep, poultry and horses, Physiol. Behav. 92 (2007) $340-374$.

[5] C.R.A. Leite-Panissi, A.A. Ferrarese, A.L.B. Terzian, L. Menescal-de-Oliveira, Serotoninergic activation of the basolateral amygdala and modulation of tonic immobility in guinea pig, Brain Res. Bull. 69 (2006) 356-364.

[6] L. De Oliveira, A. Hoffmann, L. Menescal-de-Oliveira, Participation of the medial and anterior hypothalamus in the modulation of tonic immobility in guinea pigs, Physiol. Behav. 62 (1997) 1171-1178.

[7] S. Wang, Y. Ni, F. Guo, Z. Sun, A. Ahmed, R. Zhao, Differential expression of hypothalamic fear- and stress-related genes in broiler chickens showing short or long tonic immobility, Domest. Anim. Endocrinol. 47 (2014) 65-72.

[8] X.F. de Sevilla, J. Casellas, J. Tibau, E. Fàbrega, Consistency and influence on performance of behavioural differences in Large White and Landrace purebred pigs, Appl. Anim. Behav. Sci. 117 (2009) 13-19.

[9] E. van Erp-van der Kooij, A.H. Kuijpers, J.W. Schrama, E.D. Ekkel, M.J.M. Tielen, Individual behavioural characteristics in pigs and their impact on production, Appl. Anim. Behav. Sci. 66 (2000) 171-185.

[10] H.W. Erhard, M. Mendl, Tonic immobility and emergence time in pigs-more evidence for behavioural strategies, Appl. Anim. Behav. Sci. 61 (1999) 227-237.

[11] H.W. Erhard, M. Mendl, S.B. Christiansen, Individual differences in tonic immobility may reflect behavioural strategies, Appl. Anim. Behav. Sci. 64 (1999) 31-46.

[12] M.J.C. Hessing, A.M. Hagelsø, J.A.M. van Beek, R.P. Wiepkema, W.G.P. Schouten, R. Krukow, Individual behavioural characteristics in pigs, Appl. Anim. Behav. Sci. 37 (1993) 285-295.

[13] N.. Geverink, W.G.. Schouten, G. Gort, V.. Wiegant, Individual differences in behavioral and physiological responses to restraint stress in pigs, Physiol. Behav. 77 (2002) 451-457.

[14] N.A. Geverink, M.J.W. Heetkamp, W.G.P. Schouten, V.M. Wiegant, J.W. Schrama, Backtest type and housing condition of pigs influence energy metabolism, J. Anim. Sci. 82 (2004) 1227-1233. 
[15] I. Reimert, J.E. Bolhuis, B. Kemp, T.B. Rodenburg, Social support in pigs with different coping styles, Physiol. Behav. 129 (2014) 221-229.

[16] S. Hashimoto, T. Inoue, T. Koyama, Effects of conditioned fear stress on serotonin neurotransmission and freezing behavior in rats, Eur. J. Pharmacol. 378 (1999) 23-30.

[17] T. Grandin, Assessment of stress during handling and transport, J. Anim. Sci. 75 (1997) 249-257.

[18] M. Gispert, L. Faucitano, M. Oliver, M. Guàrdia, A survey of pre-slaughter conditions, halothane gene frequency, and carcass and meat quality in five Spanish pig commercial abattoirs, Meat Sci. 55 (2000) 97-106.

[19] O. Adeola, R.O. Ball, J.D. House, P.J. O’Brien, Regional brain neurotransmitter concentrations in stress-susceptible pigs, J. Anim. Sci. 71 (1993) 968-974.

[20] S.J. Rosochacki, a. B. Piekarzewska, J. Poloszynowicz, T. Sakowski, Genetic differences in brain monoamines level in Pietrain and Duroc pigs exposed to acute restraint stress, J. Anim. Breed. Genet. 120 (2003) 192-209.

[21] R. Poletto, R.L. Meisel, B.T. Richert, H.-W. Cheng, J.N. Marchant-Forde, Aggression in replacement grower and finisher gilts fed a short-term high-tryptophan diet and the effect of long-term human-animal interaction, Appl. Anim. Behav. Sci. 122 (2010) 98110 .

[22] R. Poletto, H.W. Cheng, R.L. Meisel, J.P. Garner, B.T. Richert, J.N. Marchant-Forde, Aggressiveness and brain amine concentration in dominant and subordinate finishing pigs fed the -adrenoreceptor agonist ractopamine, J. Anim. Sci. 88 (2010) 3107-3120.

[23] R. Poletto, H.-W. Cheng, R.L. Meisel, B.T. Richert, J.N. Marchant-Forde, Gene expression of serotonin and dopamine receptors and monoamine oxidase- $\mathrm{A}$ in the brain of dominant and subordinate pubertal domestic pigs (Sus scrofa) fed a $\beta$ adrenoreceptor agonist, Brain Res. 1381 (2011) 11-20.

[24] A. Valros, P. Palander, M. Heinonen, C. Munsterhjelm, E. Brunberg, L. Keeling, P. Piepponen, Evidence for a link between tail biting and central monoamine metabolism in pigs (Sus scrofa domestica), Physiol. Behav. 143 (2015) 151-157.

[25] O. Adeola, R.O. Ball, Hypothalamic neurotransmitter concentrations and meat quality in stressed pigs offered excess dietary tryptophan and tyrosine, J. Anim. Sci. 70 (1992) 1888-1894.

[26] J. Sabria, D. Torres, M. Pasto, J.M. Peralba, A. Allali-Hassani, X. Pares, Release of neurotransmitters from rat brain nerve terminals after chronic ethanol ingestion: differential effects in cortex and hippocampus, Addict. Biol. 8 (2003) 287-294.

[27] E. Asan, M. Steinke, K.-P. Lesch, Serotonergic innervation of the amygdala: targets, receptors, and implications for stress and anxiety, Histochem. Cell Biol. 139 (2013) $785-813$.

[28] E.A. Antoniadis, R.J. McDonald, Amygdala, hippocampus and discriminative fear conditioning to context, Behav. Brain Res. 108 (2000) 1-19.

[29] P.R. Albert, F. Vahid-Ansari, C. Luckhart, Serotonin-prefrontal cortical circuitry in 
anxiety and depression phenotypes: pivotal role of pre- and post-synaptic 5-HT1A receptor expression, Front. Behav. Neurosci. 8 (2014) 199. doi: 10.3389/ fnbeh.2014.00199

[30] P. Belujon, A.A. Grace, Hippocampus, amygdala, and stress: interacting systems that affect susceptibility to addiction, Ann. N. Y. Acad. Sci. 1216 (2011) 114-121.

[31] P. Tovote, J.P. Fadok, A. Lüthi, Neuronal circuits for fear and anxiety, Nat. Rev. Neurosci. 16 (2015) 317-331.

[32] E. Vermetten, J.D. Bremner, Circuits and systems in stress. I. Preclinical studies, Depress. Anxiety. 15 (2002) 126-147.

[33] B.S. McEwen, The neurobiology of stress: from serendipity to clinical relevance, Brain Res. 886 (2000) 172-189.

[34] M. Joëls, T.Z. Baram, The neuro-symphony of stress, Nat. Rev. Neurosci. 10 (2009) 459-66.

[35] P. Belujon, A.A. Grace, Regulation of dopamine system responsivity and its adaptive and pathological response to stress, Proc. Biol. Sci. 282 (2015): 20142516.

[36] B. Olivier, Serotonin: a never-ending story, Eur. J. Pharmacol. 753 (2015) 2-18.

[37] L. Lanfumey, R. Mongeau, C. Cohen-Salmon, M. Hamon, Corticosteroid-serotonin interactions in the neurobiological mechanisms of stress-related disorders, Neurosci. Biobehav. Rev. 32 (2008) 1174-1184.

[38] T.G. Dinan, Serotonin and the regulation of hypothalamic-pituitary-adrenal axis function, Life Sci. 58 (1996) 1683-1694.

[39] R. Adolphs, The biology of fear, Curr. Biol. 23 (2013) R79-93.

[40] L.G. Kirby, A.R. Allen, I. Lucki, Regional differences in the effects of forced swimming on extracellular levels of 5-hydroxytryptamine and 5-hydroxyindoleacetic acid, Brain Res. 682 (1995) 189-196.

[41] J. Amat, P. Matus-Amat, L.R. Watkins, S.F. Maier, Escapable and inescapable stress differentially and selectively alter extracellular levels of 5-HT in the ventral hippocampus and dorsal periaqueductal gray of the rat, Brain Res. 797 (1998) 12-22.

[42] A. Vahabzadeh, M. Fillenz, Comparison of stress-induced changes in noradrenergic and serotonergic neurons in the rat hippocampus using microdialysis, Eur. J. Neurosci. 6 (1994) 1205-1212.

[43] M. Matsuo, Y. Kataoka, S. Mataki, Y. Kato, K. Oi, Conflict situation increases serotonin release in rat dorsal hippocampus: in vivo study with microdialysis and Vogel test, Neurosci. Lett. 215 (1996) 197-200.

[44] L.E. Rueter, B.L. Jacobs, A microdialysis examination of serotonin release in the rat forebrain induced by behavioral/environmental manipulations, Brain Res. 739 (1996) $57-69$.

[45] L.S. Wilkinson, T. Humby, S. Killcross, T.W. Robbins, B.J. Everitt, Dissociations in hippocampal 5-hydroxytryptamine release in the rat following Pavlovian aversive 
conditioning to discrete and contextual stimuli, Eur. J. Neurosci. 8 (1996) 1479-1487.

[46] E. Hajos-Korcsok, D. Robinson, J. Yu, C. Fitch, Rapid habituation of hippocampal serotonin and norepinephrine release and anxiety-related behaviors, but not plasma corticosterone levels, to repeated footshock, Pharmacology, Biochemistry and Behavior 74 (2003) 609-616.

[47] F. Fraisse, J.F. Cockrem, Corticosterone and fear behaviour in white and brown caged laying hens, Br. Poult. Sci. 47 (2006) 110-119.

[48] U. Stockhorst, M.I. Antov, Modulation of Fear Extinction by Stress, Stress Hormones and Estradiol: A Review, Front. Behav. Neurosci. 9 (2015) 359. doi: 10.3389/fnbeh.2015.00359

[49] A. Boissy, Fear and fearfulness in animals, Q. Rev. Biol. 70 (1995) 165-171.

[50] W.W. Ursinus, J.E. Bolhuis, J.J. Zonderland, T.B. Rodenburg, A.S. de Souza, R.E. Koopmanschap, B. Kemp, G.A.H. Korte-Bouws, S.M. Korte, C.G. van Reenen, Relations between peripheral and brain serotonin measures and behavioural responses in a novelty test in pigs, Physiol. Behav. 118 (2013) 88-96.

[51] R.L. Dennis, D.C. Lay, H.W. Cheng, Effects of early serotonin programming on behavior and central monoamine concentrations in an avian model, Behav. Brain Res. 253 (2013) 290-296.

[52] S. Korte, S. De Boer, A robust animal model of state anxiety: fear-potentiated behaviour in the elevated plus-maze, Eur. J. Pharmacol. 463 (2003) 163-175.

[53] K. Tada, K. Kasamo, T. Suzuki, Y. Matsuzaki, Endogenous 5-HT inhibits firing activity of hippocampal CA1 pyramidal neurons during conditioned fear stress-induced freezing behavior through stimulating 5-HT1A, Hippocampus 14 (2004) 143-147.

[54] C. Gross, L. Santarelli, D. Brunner, X. Zhuang, R. Hen, Altered fear circuits in 5-HT 1A receptor KO mice, Biol. Psychiatry 48 (2000) 1157-1163.

[55] B. Westerink, Brain microdialysis and its application for the study of animal behaviour, Behav. Brain Res. 70 (1995) 103-124.

[56] S. Puglisi-Allegra, D. Andolina, Serotonin and stress coping, Behav. Brain Res. 277 (2015) 58-67.

[57] M. Hale, A. Shekhar, C. Lowry, Development by environment interactions controlling tryptophan hydroxylase expression, J. Chem. Neuroanat. 41 (2011) 219-226.

[58] L. Gutknecht, C. Kriegebaum, J. Waider, A. Schmitt, Spatio-temporal expression of tryptophan hydroxylase isoforms in murine and human brain: convergent data from Tph2 knockout mice, Eur. Neuropsychopharmacol. 19 (2009) 266-282.

[59] S.C. Daubner, T. Le, S. Wang, Tyrosine hydroxylase and regulation of dopamine synthesis, Arch. Biochem. Biophys. 508 (2011) 1-12.

[60] E. Sabban, R. Kvetňanský, Stress-triggered activation of gene expression in catecholaminergic systems: dynamics of transcriptional events, Trends Neurosci. 24 (2001) 91-98. 
[61] I. Winge, J.A. McKinney, M. Ying, C.S. D’Santos, R. Kleppe, P.M. Knappskog, J. Haavik, Activation and stabilization of human tryptophan hydroxylase 2 by phosphorylation and 14-3-3 binding, Biochem. J. 410 (2008) 195-204.

[62] D.M. Kuhn, S.A. Sakowski, T.J. Geddes, C. Wilkerson, J.W. Haycock, Phosphorylation and activation of tryptophan hydroxylase 2: identification of serine-19 as the substrate site for calcium, calmodulin-dependent protein kinase II, J. Neurochem. 103 (2007) 1567-1573.

[63] N. Carkaci-Salli, U. Salli, I. Tekin, J.A. Hengst, M.K. Zhao, T.L. Gilman, A.M. Andrews, K.E. Vrana, Functional characterization of the S41Y (C2755A) polymorphism of tryptophan hydroxylase 2, J. Neurochem. 130 (2014) 748-758. 
Table 1. Concentration of neurotransmitters (ng/g tissue) in brain areas

\begin{tabular}{|c|c|c|c|c|c|c|c|c|}
\hline & \multicolumn{2}{|c|}{ Amygdala } & \multicolumn{2}{|c|}{ PFC } & \multicolumn{2}{|c|}{ Hippocampus } & \multicolumn{2}{|c|}{ Hypothalamus } \\
\hline & Mean & $\mathrm{SE}$ & Mean & $\mathrm{SE}$ & Mean & $\mathrm{SE}$ & Mean & $\mathrm{SE}$ \\
\hline $\mathrm{NA}$ & 152.50 & 9.37 & 144.39 & 4.00 & 130.48 & 5.03 & 2007.19 & 83.77 \\
\hline L-DOPA & - & - & - & - & - & - & 309.91 & 18.73 \\
\hline DOPAC & 43.65 & 4.04 & 7.28 & 0.86 & - & - & 59.40 & 7.19 \\
\hline DA & 359.69 & 24.42 & 19.95 & 1.31 & 27.52 & 2.63 & 302.27 & 15.05 \\
\hline HVA & 280.27 & 18.39 & 61.45 & 4.93 & - & - & 295.61 & 22.09 \\
\hline Total Catecholamines & 860.48 & 40.86 & 232.62 & 11.13 & 154.81 & 5.31 & 2911.25 & 103.77 \\
\hline 5-HIAA & 270.97 & 13.22 & 111.10 & 5.16 & 129.74 & 5.41 & 373.24 & 23.09 \\
\hline $5-\mathrm{HT}$ & 917.38 & 47.35 & 252.71 & 10.79 & 276.56 & 9.83 & 995.47 & 51.79 \\
\hline Total Indoleamines & 1188.35 & 58.29 & 363.81 & 14.66 & 406.30 & 13.65 & 1368.71 & 70.88 \\
\hline 5- HIAA/5-HT & 0.31 & 0.01 & 0.45 & 0.02 & 0.48 & 0.02 & 0.08 & 0.02 \\
\hline DOPAC/DA & 0.11 & 0.01 & 0.37 & 0.04 & - & - & 0.19 & 0.02 \\
\hline HVA/DA & 0.91 & 0.09 & 3.34 & 0.24 & - & - & 1.03 & 0.09 \\
\hline NA/DA & 0.83 & 0.38 & 8.39 & 0.63 & 6.55 & 0.91 & 6.94 & 0.33 \\
\hline$(\mathrm{DOPAC}+\mathrm{HVA}) / \mathrm{DA}$ & 0.97 & 0.04 & 3.83 & 0.27 & - & - & 1.28 & 0.12 \\
\hline DOPAC+HVA & 330.88 & 19.84 & 67.41 & 5.81 & - & - & 335.77 & 26.89 \\
\hline Indoleamines/Catecholamines & 1.452 & 0.05 & 1.79 & 0.12 & 2.68 & 0.126 & 0.48 & 0.02 \\
\hline
\end{tabular}


Table 2. Eigenvalues, individual and cumulative proportion of the Correlation Matrix and loadings of neurotransmitters in varimax rotated factor matrix. Loadings equals or higher than 0.50 are highlighted to indicate the main attributes of the different principal components (PC).

\begin{tabular}{lcc}
\hline & PC1 & PC2 \\
\hline Eigenvalue & 5.053 & 1.395 \\
Individual Proportion & $72.19 \%$ & $19.92 \%$ \\
Cumulative Proportion & $72.19 \%$ & $92.11 \%$ \\
\hline Variables & & \\
NA & 0.23 & $\mathbf{0 . 9 7}$ \\
L-DOPA & 0.22 & $\mathbf{0 . 9 7}$ \\
DOPAC & $\mathbf{0 . 9 0}$ & 0.28 \\
DA & $\mathbf{0 . 9 5}$ & 0.11 \\
HVA & $\mathbf{0 . 9 3}$ & 0.18 \\
5-HIAA & $\mathbf{0 . 8 2}$ & 0.46 \\
5-HT & $\mathbf{0 . 9 1}$ & 0.26 \\
\hline
\end{tabular}


Table 3. Effects of stressful handling at the slaughterhouse on the neurotransmitter concentration (ng/g tissue) in amygdala, PFC, hippocampus and hypothalamus in pigs. $* P<0.05$

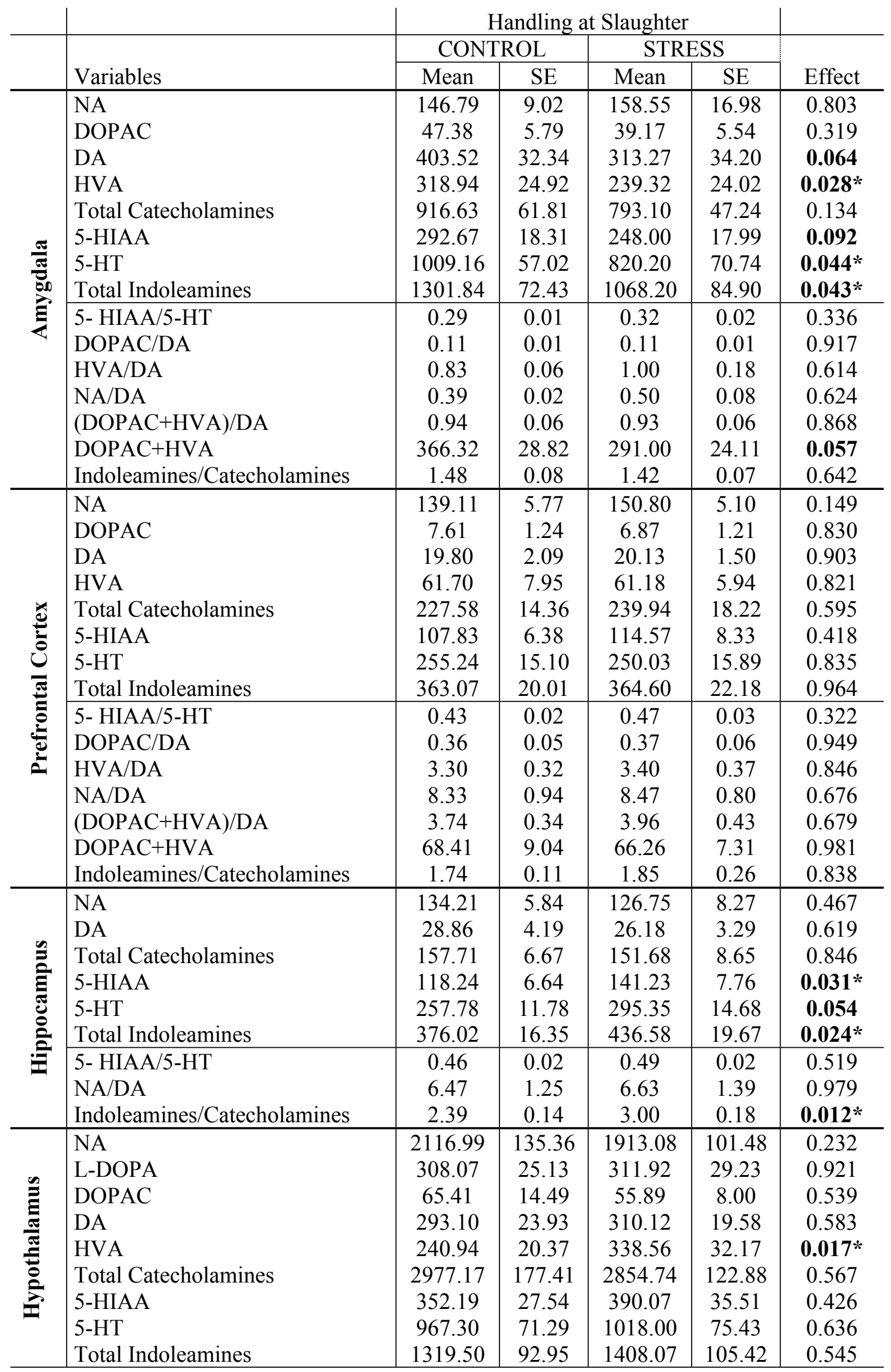




\begin{tabular}{l|c|c|c|c|c}
\hline 5- HIAA/5-HT & 0.37 & 0.02 & 0.39 & 0.02 & 0.729 \\
DOPAC/DA & 0.21 & 0.06 & 0.18 & 0.02 & 0.781 \\
HVA/DA & 0.86 & 0.09 & 1.16 & 0.14 & 0.108 \\
NA/DA & 7.47 & 0.44 & 6.48 & 0.46 & 0.343 \\
(DOPAC+HVA)/DA & 1.02 & 0.14 & 1.41 & 0.16 & 0.132 \\
DOPAC+HVA & 271.25 & 28.51 & 386.47 & 37.98 & $\mathbf{0 . 0 2 0 *}$ \\
Indoleamines/Catecholamines & 0.45 & 0.02 & 0.50 & 0.04 & 0.220 \\
\hline
\end{tabular}


Table 4. Neurotransmitter concentration (ng/g tissue) in amygdala, PFC, hippocampus and hypothalamus in pigs and the influence of the TI test in the response to control or stressful handling at the slaughterhouse. ${ }^{*} P<0.05$

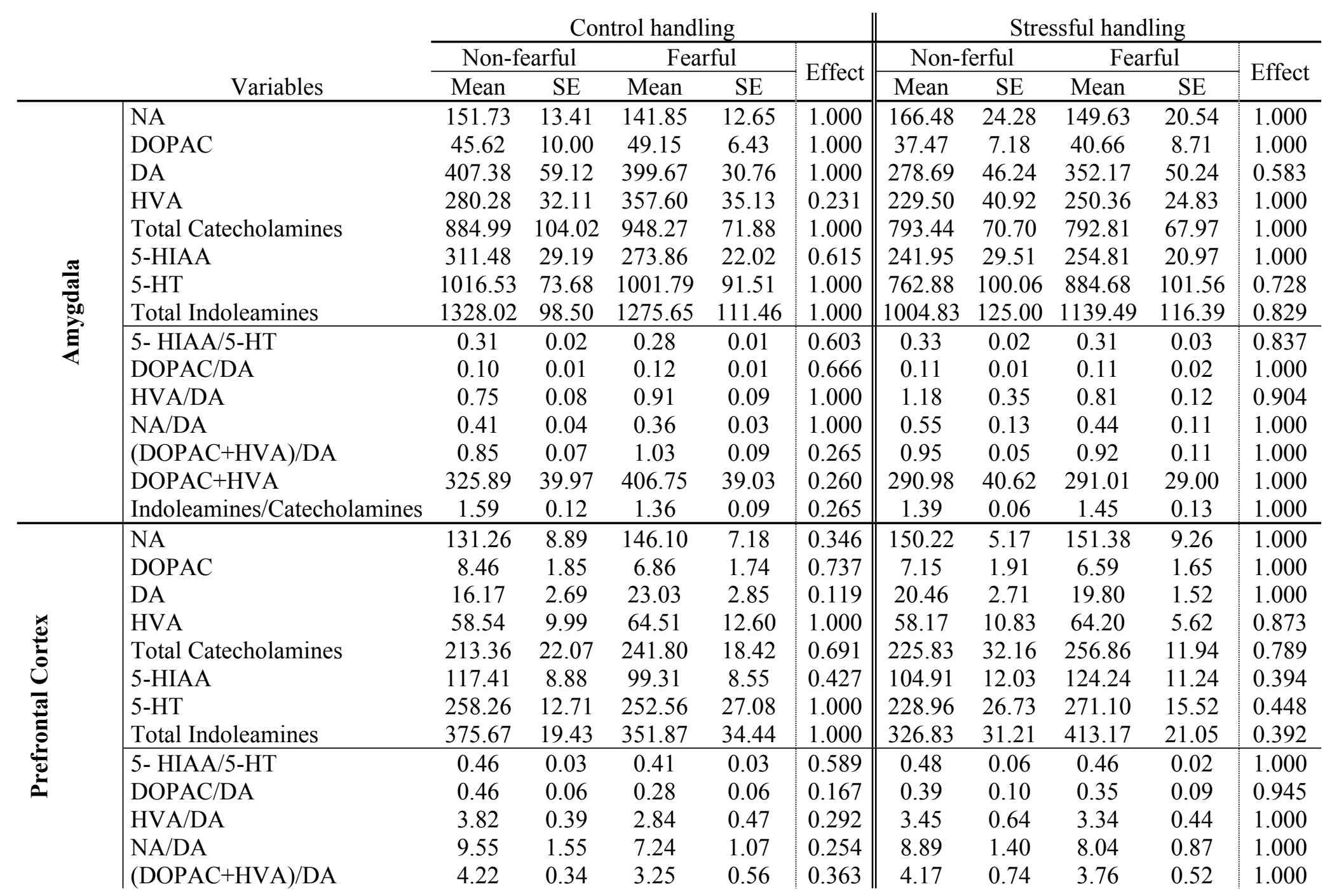




\begin{tabular}{|c|c|c|c|c|c|c|c|c|c|c|c|}
\hline & $\begin{array}{l}\text { DOPAC }+ \text { HVA } \\
\text { Indoleamines/Catecholamines }\end{array}$ & $\begin{array}{c}65.94 \\
1.87\end{array}$ & $\begin{array}{c}11.74 \\
0.19\end{array}$ & $\begin{array}{l}70.61 \\
1.61\end{array}$ & $\begin{array}{c}14.16 \\
0.12\end{array}$ & $\begin{array}{l}1.000 \\
1.000\end{array}$ & $\begin{array}{c}63.54 \\
1.96\end{array}$ & $\begin{array}{c}12.59 \\
0.48\end{array}$ & $\begin{array}{c}69.38 \\
1.72\end{array}$ & $\begin{array}{l}7.26 \\
0.13\end{array}$ & $\begin{array}{l}0.968 \\
1.000\end{array}$ \\
\hline \multirow{9}{*}{ 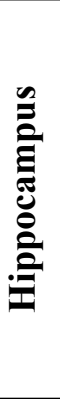 } & NA & 140.31 & 7.64 & 128.79 & 8.71 & 0.874 & 123.84 & 11.01 & 130.03 & 13.16 & 1.000 \\
\hline & $\mathrm{DA}$ & 33.01 & 6.68 & 24.72 & 5.09 & 0.566 & 23.31 & 4.61 & 30.00 & 4.57 & 0.777 \\
\hline & Total Catecholamines & 170.51 & 9.61 & 144.92 & 6.80 & 0.128 & 136.35 & 7.05 & 169.56 & 14.17 & $0.047 *$ \\
\hline & 5-HIAA & 119.86 & 10.72 & 116.81 & 8.75 & 1.000 & 134.88 & 8.42 & 148.38 & 13.70 & 0.733 \\
\hline & 5-HT & 253.05 & 21.67 & 261.98 & 12.42 & 1.000 & 265.13 & 13.90 & 329.35 & 22.04 & $0.030 *$ \\
\hline & Total Indoleamines & 372.91 & 30.80 & 378.79 & 16.41 & 1.000 & 400.01 & 20.11 & 477.73 & 30.03 & 0.063 \\
\hline & 5- HIAA/5-HT & 0.48 & 0.03 & 0.45 & 0.04 & 1.000 & 0.51 & 0.03 & 0.46 & 0.04 & 0.537 \\
\hline & NA/DA & 5.76 & 1.44 & 7.18 & 2.11 & 1.000 & 7.09 & 2.13 & 6.09 & 1.91 & 1.000 \\
\hline & Indoleamines/Catecholamines & 2.15 & 0.19 & 2.62 & 0.16 & 0.290 & 3.09 & 0.27 & 2.88 & 0.26 & 1.000 \\
\hline \multirow{16}{*}{ 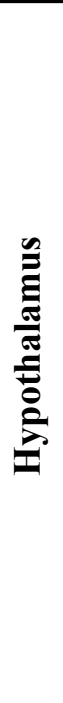 } & $\mathrm{NA}$ & 2011.75 & 177.09 & 2264.34 & 213.90 & 0.613 & 1724.17 & 128.95 & 2054.75 & 133.69 & 0.303 \\
\hline & L-DOPA & 295.22 & 37.60 & 326.05 & 32.57 & 1.000 & 238.27 & 29.48 & 354.01 & 34.31 & 0.090 \\
\hline & DOPAC & 58.26 & 16.61 & 74.94 & 28.97 & 1.000 & 57.50 & 13.78 & 54.27 & 9.52 & 1.000 \\
\hline & $\mathrm{DA}$ & 293.43 & 41.27 & 292.63 & 14.28 & 1.000 & 286.96 & 35.20 & 327.50 & 21.95 & 0.713 \\
\hline & HVA & 224.42 & 20.66 & 269.85 & 43.28 & 0.704 & 397.28 & 58.88 & 294.52 & 29.32 & 0.202 \\
\hline & Total Catecholamines & 2858.10 & 254.02 & 3143.87 & 245.60 & 0.729 & 2624.76 & 72.66 & 3027.23 & 190.60 & 0.343 \\
\hline & 5-HIAA & 327.84 & 30.67 & 386.30 & 50.30 & 0.859 & 383.88 & 47.08 & 395.49 & 55.28 & 1.000 \\
\hline & 5-HT & 906.42 & 101.21 & 1052.53 & 94.46 & 0.714 & 900.42 & 114.09 & 1120.88 & 91.29 & 0.245 \\
\hline & Total Indoleamines & 1234.26 & 128.72 & 1438.83 & 126.98 & 0.710 & 1284.30 & 155.95 & 1516.37 & 140.86 & 0.476 \\
\hline & 5- HIAA/5-HT & 0.38 & 0.03 & 0.37 & 0.04 & 1.000 & 0.43 & 0.02 & 0.35 & 0.03 & 0.120 \\
\hline & DOPAC/DA & 0.16 & 0.03 & 0.28 & 0.13 & 0.842 & 0.19 & 0.03 & 0.16 & 0.03 & 1.000 \\
\hline & HVA/DA & 0.85 & 0.13 & 0.88 & 0.13 & 1.000 & 1.45 & 0.25 & 0.94 & 0.13 & 0.096 \\
\hline & NA/DA & 7.26 & 0.63 & 7.76 & 0.65 & 1.000 & 6.59 & 0.96 & 6.41 & 0.45 & 1.000 \\
\hline & $(\mathrm{DOPAC}+\mathrm{HVA}) / \mathrm{DA}$ & 0.95 & 0.16 & 1.18 & 0.32 & 0.959 & 1.64 & 0.26 & 1.17 & 0.17 & 0.261 \\
\hline & DOPAC+HVA & 257.71 & 31.60 & 294.94 & 60.51 & 1.000 & 454.78 & 67.95 & 335.23 & 36.59 & 0.270 \\
\hline & Indoleamines/Catecholamines & 0.44 & 0.04 & 0.46 & 0.03 & 1.000 & 0.50 & 0.07 & 0.51 & 0.04 & 1.000 \\
\hline
\end{tabular}




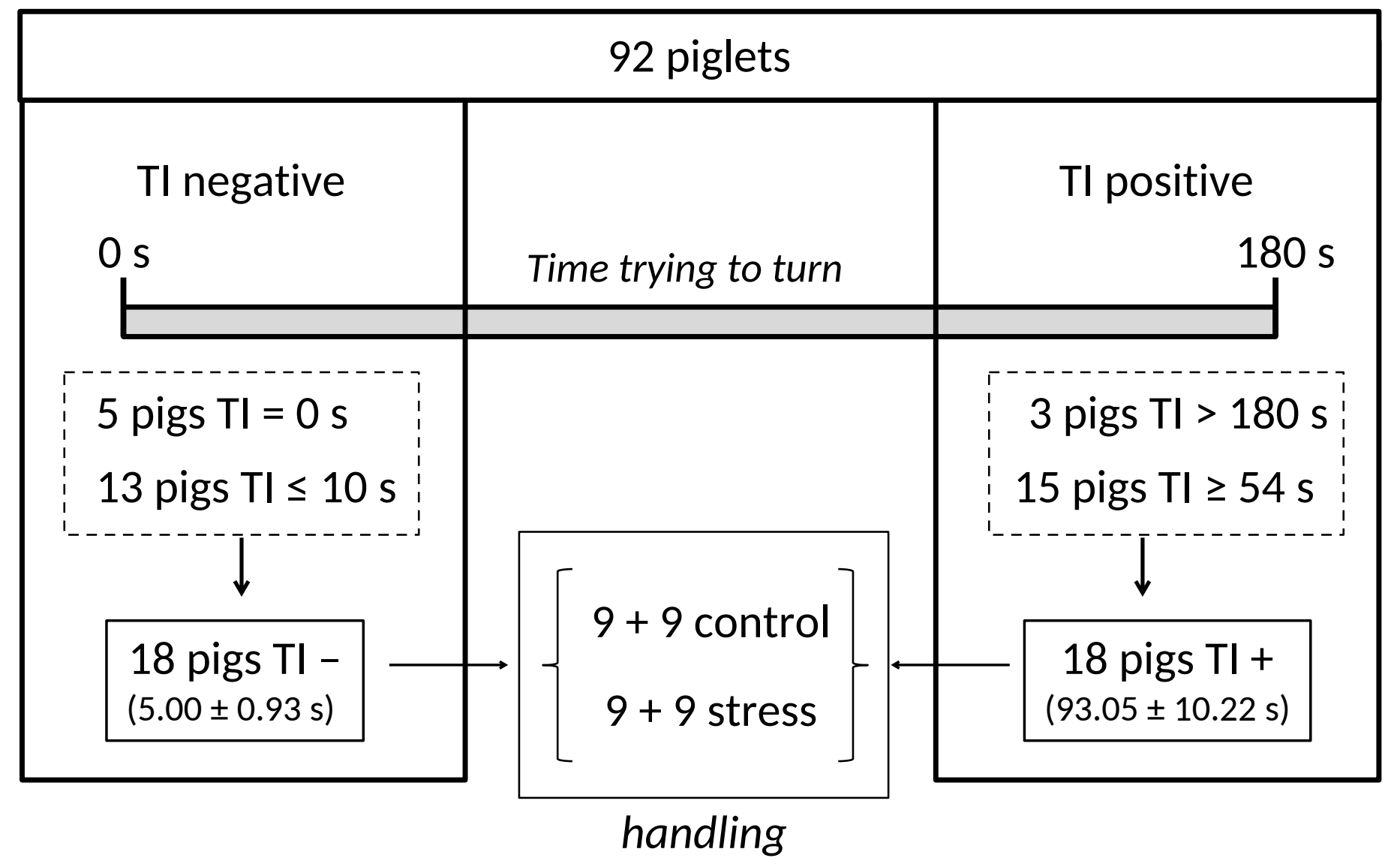

Figure 1 


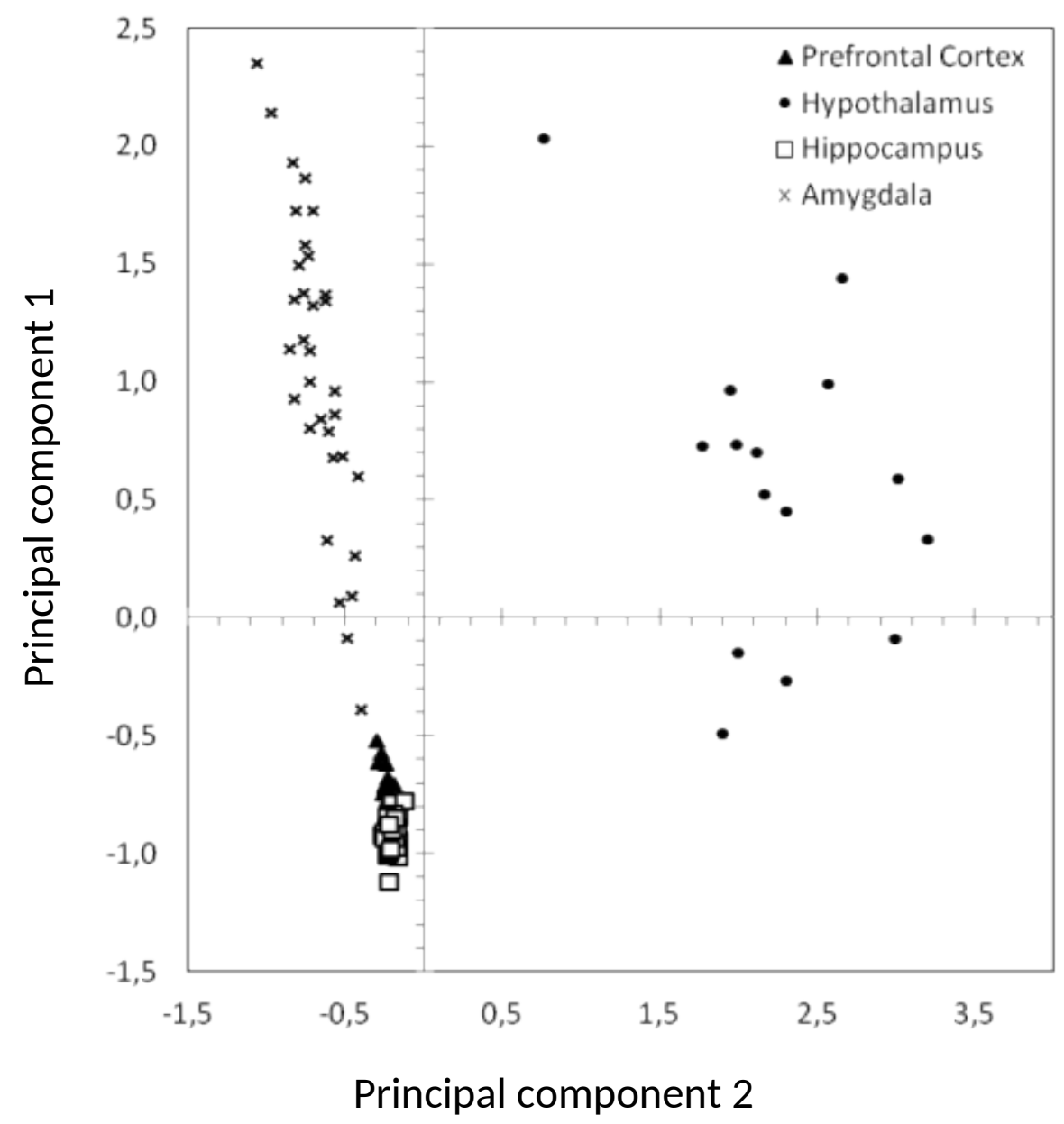

Figure 2 


\section{Highlights}

- We measured brain neurotransmitters in pigs classified as fearful-nonfearful under stressful handling.

- Stressful handling alters the 5-HT system in the hippocampus and the amygdala.

- There was no difference between fearful and non-fearful pigs under non-stressful handling.

- The 5-HT pathway is activated in the hippocampus under stressful handling only in fearful pigs. 